Conclusion: In early arthritis patients, during 5 years treated to target drug free DAS-remission, disease flares with loss of DAS-remission were common. Although the majority of patients who flared were still in LDA, most reported more pain, morning stiffness, increased disease activity and a diminished global health. On average, deterioration in HAQ only exceeded the minimum clinically important difference (delta $H A Q>=0.22$ ) in case of $a \geq 0.6$ increase in DAS, independent of the previous DAS. Depending on the definition of flare, up to $45 \%$ of patients lost DAS LDA, and in this group the functional deterioration significantly more often exceeded the MCID as compared to the patients that flared but were still in LDA. More research is needed to find out which patients are most at risk for clinically relevant flares, and to evaluate the impact of flares in patients with remission on long term outcomes.

Table 1. Odds Ratios and $95 \%$ confidence intervals for $>20 \mathrm{~mm}$ increase in PROs on $100 \mathrm{~mm}$ visual analogue scales

\begin{tabular}{|c|c|c|c|c|c|c|}
\hline & \multicolumn{2}{|l|}{ Flare A } & \multicolumn{2}{|c|}{ Flare B (minor) } & \multicolumn{2}{|c|}{ Flare C (major) } \\
\hline & $\begin{array}{l}\text { Prevalence } \\
\geq 20 \mathrm{~mm}^{\mathrm{a}}\end{array}$ & $\begin{array}{l}\text { OR } \\
(95 \% \mathrm{Cl})\end{array}$ & $\begin{array}{l}\text { Prevalence } \\
\geq 20 \mathrm{~mm}^{\mathrm{a}}\end{array}$ & $\begin{array}{l}\text { OR } \\
(95 \% \mathrm{Cl})\end{array}$ & $\begin{array}{l}\text { Prevalence } \\
\geq 20 \mathrm{~mm}^{\mathrm{a}}\end{array}$ & $\begin{array}{l}\text { OR } \\
(95 \% \mathrm{Cl})\end{array}$ \\
\hline Global health & $62 \%$ & $2.1(1.5 ; 2.8)$ & $45 \%$ & $\begin{array}{l}0.5(0.4 \\
0.7)\end{array}$ & $62 \%$ & $1.4(1.1 ; 1.8)$ \\
\hline Disease activity & $62 \%$ & $2.5(1.7 ; 3.8)$ & $45 \%$ & $\begin{array}{l}0.4(0.3 \\
0.6)\end{array}$ & $62 \%$ & $\begin{array}{l}2.1(1.4 ; \\
3.0)\end{array}$ \\
\hline Pain & $87 \%$ & $2.0(1.3 ; 3.1)$ & $78 \%$ & $\begin{array}{l}0.5(0.3 \\
0.8)\end{array}$ & $87 \%$ & $1.8(1.2 ; 2.5)$ \\
\hline Morning stiffness & $84 \%$ & $1.7(1.1 ; 2.6)$ & $77 \%$ & $\begin{array}{l}0.6(0.4 \\
0.9)\end{array}$ & $86 \%$ & $\begin{array}{l}2.1 \text { (1.5; } \\
2.9)\end{array}$ \\
\hline
\end{tabular}

The prevalence of $>20 \mathrm{~mm}$ deterioration in VAS PRO's during a visit with a flare.

Acknowledgements: We would like to thank all patients for their contribution as well as the rheumatologists who participated in the IMPROVED-study group. We would also like to thank all other rheumatologists and trainee rheumatologists who enrolled patients in these studies, and all research nurses for their contributions.

Disclosure of Interests: Johanna M. Maassen: None declared, Sytske Anne Bergstra: None declared, Petronella DM de Buck: None declared, M. van Oosterhout: None declared, Thomas Huizinga: None declared, Cornelia Allaart Grant/ research support from: the IMPROVED study was designed by the investigators and financially supported by AbbVie in the first year.

DOI: 10.1136/annrheumdis-2021-eular.2656

\section{POS0480 ASSOCIATION OF NEUTROPHIL LYMPHOCYTE AND PLATELET LYMPHOCYTE RATIOS WITH JOINT INFLAMMATION IN RHEUMATOID ARTHRITIS}

D. Pearce-Fisher ${ }^{1}$, D. Orange ${ }^{1,2}$, B. Mehta ${ }^{1,3}$, D. Jannat-Khah ${ }^{1,3}$, S. Goodman ${ }^{1,3}$. ${ }^{1}$ Hospital for Special Surgery, Medicine, New York, United States of America;

${ }^{2}$ The Rockefeller University, Clinical Investigation, New York, United States of America; ${ }^{3}$ Weill Cornell Medicine, Medicine, New York, United States of America

Background: Some patients with rheumatoid arthritis (RA) have high disease activity scores (DAS) and low synovial inflammation, and others have high synovial inflammation and low DAS (subclinical synovitis)[1]. It would be clinically useful to identify blood biomarkers of synovial inflammation. Neutrophil-lymphocyte (NLR) and platelet-lymphocyte ratios (PLR) have been reported to distinguish RA patients with moderate/high DAS28 scores from low DAS28 [2]. However, it is not known if these inexpensive, accessible tests are associated with inflammation in synovial tissue at the histological level.

Objectives: The objective of this study was to evaluate the relationship of pre-operative NLR and PLR with synovial inflammation of the operative joint in RA patients undergoing arthroplasty.

Methods: 230 patients meeting ACR/EULAR 1987 and/or 2010 criteria were recruited prior to elective total hip, knee, shoulder, and elbow replacement. Demographics, RA characteristics, medications, disease activity, and routine tests including complete blood tests $(\mathrm{CBC})$ were collected pre-operatively. Hematoxylin and eosin (H\&E) stains were prepared from the synovium of the operative joint and systematically scored by a pathologist as described previously [3]. Synovial lymphocytic inflammation was graded as none, mild, moderate, marked, or band-like. Linear regression was performed to distinguish differences in the NLR, PLR, and CRP in patients with synovial lymphocytic inflammation (SLI).
Results: As expected, patients on glucocorticoids (GCs) had higher NLR (mean 5.52 (SD 7.68) vs mean 2.82 (SD 1.66) $(\mathrm{p}<0.001)$ and higher PLR (mean 233.73 (SD 237.21) vs (mean 162.93 (SD 65.35)) (p-value=0.04)) and those patients $(\mathrm{N}=92)$ were therefore excluded from down-stream analyses. On the remaining 138 patients, we tested for associations of PLR, NLR and CRP with SLI using linear regression. In all the models the highest category for synovial lymphocytic inflammation was found to be statistically significantly associated with NLR, PLR and CRP, separately (Table 1).

Table 1. Results from linear regressions evaluating the association of NLR, PLR, and CRP with synovial lymphocytic inflammation.

\begin{tabular}{llll}
\hline Linear regression Results & NLR & PLR & CRP \\
\hline Synovial Lymphocytic Inflammation Coef $(95 \% \mathrm{Cl})$ & Coef $(95 \% \mathrm{Cl})$ & Coef $(95 \% \mathrm{Cl})$ \\
\hline None & Reference & reference & reference \\
Mild & $0.31(-0.51,1.13)$ & $26.54(-8.83,61.90)$ & $-1.00(-2.37,0.36)$ \\
Moderate & $0.73(-0.18,1.64)$ & $28.66(-10.22,67.53)$ & $0.46(-1.09,2.01)$ \\
Marked & $0.21(-0.80,1.22)$ & $24.62(-22.80,72.05)$ & $0.81(-0.87,2.49)$ \\
Band-like & $\mathbf{1 . 9 2}(\mathbf{0 . 8 1}, \mathbf{3 . 0 2})$ & $\mathbf{8 0 . 4 2}(\mathbf{3 1 . 4 6 , 1 2 9 . 3 8})$ & $\mathbf{2 . 3 2}(\mathbf{0 . 4 9 , 4 . 1 6 )}$ \\
\end{tabular}

OR= Odds ratio, Coef $=$ Coefficient, NLR= neutrophil lymphocyte, $\mathrm{PLR}=$ platelet lymphocyte ratio, $\mathrm{CRP}=\mathrm{C}$-reactive proteinAll significant associations are bolded.

Conclusion: NLR, PLR and CRP are associated with high synovial lymphocytic inflammation of the operative joint. This suggests that these inexpensive, routinely performed blood tests may be a useful blood biomarker of synovial inflammation.

REFERENCES:

[1] Orange, D.E. et al. Histologic and Transcriptional Evidence of Subclinical Synovial Inflammation in Patients With Rheumatoid Arthritis in Clinical Remission. Arthritis Rheumatol. 71(7): 1034-1041 (2019).

[2] Lee, Y.H. Association between the Neutrophil-to-lymphocyte Ratio, and Platelet-to-lymphocyte Ratio and Rheumatoid Arthritis and their Correlations with the Disease Activity: A Meta-analysis. J Rheum Dis. 25(3):169-178 (2018).

[3] Orange, D. E. et al. Identification of Three Rheumatoid Arthritis Disease Subtypes by Machine Learning Integration of Synovial Histologic Features and RNA Sequencing Data. Arthritis Rheumatol. Hoboken NJ 70: 690-701 (2018).

Disclosure of Interests: Diyu Pearce-Fisher: None declared, Dana Orange Consultant of: Astra Zeneca/Medlmmune and Pfizer, Bella Mehta Consultant of: Novartis, Deanna Jannat-Khah: None declared, Susan Goodman Consultant of: UCB, Grant/research support from: Novartis, Horizon Pharmaceuticals

DOI: 10.1136/annrheumdis-2021-eular.2729

\section{POS0481 DOES OLDER REALLY MEAN WISER?}

D. Khalifa ${ }^{1}$, S. Belghali ${ }^{1}$, M. Limam ${ }^{2}$, N. El Amri ${ }^{1}$, K. Baccouche ${ }^{1}$, H. Zeglaoui ${ }^{1}$ E. Bouajina ${ }^{1} .{ }^{1}$ Farhat Hached Hospital, Rheumatology, Sousse, Tunisia; ${ }^{2}$ Farhat Hached Hospital, Preventive Medicine, Sousse, Tunisia

Background: One of the main challenges in Rheumatoid arthritis (RA) is to maintain remission or low disease activity by adhering to the prescribed treatments. However, it is believed that adherence to long term treatments is inadequate in chronic diseases. Does this apply to older people too?

Objectives: To assess treatment adherence among an elderly RA population and to study determining factors of non-adherence

Methods: A cross-sectional study over a period of 4 months was conducted in a rheumatology clinic (September 2020-December 2020). Consenting elderly over the age of 65 presenting with RA were included. Exclusion criteria involved associated connective tissue diseases and troubles communicating. During clinical visits, sociodemographic information, clinical, radiologica and therapeutic data were collected. Treatment adherence was assessed by the 5-item compliance questionnaire of rheumatology (CQR-5). Patients were also asked about the degree of satisfaction vis-à-vis the therapeutic effect detected. Univariate and multivariate analysis were conducted using the statistical tool SPSS.20.

Results: Forty patients consented to join the study, $82.5 \%$ of them were women. The median of age was 66.5 years old with a minimum of 65 and a maximum of 83 . Comorbidities were noted in $55 \%$ of the patients. $75 \%$ of the patients had health insurance. The mean pain score was 5 out of 10 on a visual analog scale (VAS). The mean DAS 28 was $4.65 \pm 1.77$ with $72.5 \%$ 
of the patients having an active disease. Deformities were reported in $60 \%$ of the cases and bone destruction on radiographs in $87.2 \%$ of the cases. Methotrexate was the most prescribed treatment in $95 \%$ of the cases and biologics in $12.5 \%$ of the cases. The median number of pills taken (on the day of MTX) was 12.5 ( $\min =6$; $\max =23$ ). Up to $35.5 \%$ of the patients were not satisfied with treatment results. $90 \%$ of the patients took their medication because they had fewer problems afterwards. $72.5 \%$ of them did not dare to miss their RA treatment. $95 \%$ of them had their pills stored in the same place to not forget them, $97.5 \%$ took their medication because they had complete confidence in their rheumatologist and $92.5 \%$ of them hung on to what the doctor told them. The overall results of the CQR-5 revealed that $72.5 \%$ of the patients were adherent to their RA medication and $27.5 \%$ of them were non-adherent. The study of factors associated to non-adherence showed that being younger $(p=0.01)$, not having a health insurance $(p=0.014)$, having comorbidities (0.03), and not being satisfied with treatments' results $(p=0.014)$ were associated to non-adherence. Multivariate analysis showed that risk factors associated to non-adherence were elevated pain scores (odds ratio $=4.11, p=0.042$ ), not having health insurance (odd ratio $=7.54, \mathrm{p}=0.006$ ) and having to take many pills at once (odds ratio $=4.48, p=0.034)$. Being older was a protector factor of non-adherence (odds ratio $=0.81, p=0.034$ )

Conclusion: Adherence among RA elderly patients is optimal and they have good trust in their medication and confidence in their rheumatologist. Older seems wiser when it comes to treatment taking behaviors in RA.

\section{REFERENCES:}

[1] Hughes LD, Done J, Young A. A 5 item version of the Compliance Questionnaire for Rheumatology (CQR5) successfully identifies low adherence to DMARDs. BMC Musculoskelet Disord 2013; 14: 286

Disclosure of Interests: None declared

DOI: 10.1136/annrheumdis-2021-eular.2738

\section{POS0482 WOOD SMOKE EXPOSURE IS ASSOCIATED WITH HIGHER ANTI-CCP ANTIBODY TITERS IN HISPANIC PATIENTS WITH RHEUMATOID ARTHRITIS}

A. B. Rodriguez-Romero ${ }^{1}$, D. Á. Galarza-Delgado ${ }^{1}$, J. R. Azpiri-López ${ }^{2}$, I. J. Colunga-Pedraza ${ }^{1}$, N. Guajardo-Jauregui ${ }^{1}$, S. Lugo-Perez ${ }^{2}$, A. Cárdenas ${ }^{1}$. ${ }^{1}$ Hospital Universitario “Dr. José Eleuterio González”, Universidad Autónoma de Nuevo León, Rheumatology Service, Monterrey, Mexico; ${ }^{2}$ Hospital Universitario "Dr. José Eleuterio González", Universidad Autónoma de Nuevo León, Cardiology Service, Monterrey, Mexico

Background: Wood smoke exposure is a risk factor for the development of chronic obstructive pulmonary disease (COPD), lung cancer and cardiovascular disease and it has also been linked to higher anti-CCP antibodies in patients with rheumatoid arthritis (RA) and COPD (1).

Objectives: The objective of the present study is to report the correlation between anti-CCP, IgG, IgM and IgA rheumatoid factor (RF) to wood smoke exposure in patients with RA. Additionally, evaluate the impact of disease activity, biomass exposure, and disease duration on anti-CCP antibody levels.

Methods: A cross-sectional, observational study was designed based on a cohort of Hispanic RA patients. All fulfilled the 2010 ACR/EULAR classification criteria for RA. Biomass smoke exposure was expressed using the biomass exposure index (BEI) calculated from the mean of exposed hours per day multiplied by the number of years exposed. Subjects were divided into two groups: those exposed to wood smoke with $\mathrm{BEI} \geq 1$ and subjects not exposed to wood smoke. They were matched by age, gender, and comorbidities. AntiCCP antibodies and RF were measured by ELISA with cutoff points of $<5 \mathrm{U}$ / $\mathrm{mL}$ and $<20 \mathrm{U} / \mathrm{mL}$ respectively.

Results: A total of 318 subjects were included, $159(50 \%)$ of them had a history of exposure to wood smoke. Anti-CCP antibody positivity was present in $102(64.2 \%)$ with a median titer of $97.1 \mathrm{U} / \mathrm{mL}(1.7-198)$ in the RA exposed group, and in $89(56 \%)$ with a median titer of $8.5 \mathrm{U} / \mathrm{mL}(1.1-145)$ in the RA non-exposed group. A significant difference was found in anti-CCP antibody titers between groups $(p=0.003)$. (Table 1$)$. Spearman's rho showed a small but statistically significant correlation between BEI and anti-CCP antibody titers ( $r h o=0.170, p=0.002$ ). Biomass exposure was independently related to higher anti-CCP antibody titers $(B=35.4, p<0.001)$.

Conclusion: RA patients who were exposed to wood smoke had higher titers of anti-CCP antibodies than non-exposed RA patients. Furthermore, biomass exposure was shown to be independently related to higher titers of anti-CCP antibodies.
Table 1. Comparison of demographic, seropositivity and clinical characteristic between patients with RA exposed and matched non-exposed RA patients.

\begin{tabular}{|c|c|c|c|}
\hline Characteristics & $\begin{array}{l}\text { RA exposed } \\
(n=159)\end{array}$ & $\begin{array}{l}\text { RA not exposed } \\
(n=159)\end{array}$ & $\mathbf{p}$ \\
\hline Age years, \pm SD & $56.7 \pm 8.7$ & $55.4 \pm 8.1$ & NS \\
\hline Female, $\mathrm{n}(\%)$ & $148(93.1)$ & $148(93.1)$ & NS \\
\hline BEI years, median (p25-p75) & $35(15-90)$ & 0 & \\
\hline Disease duration years, median (p25-p75) & $9(3.5-15.1)$ & $6.8(2.8-14.6)$ & NS \\
\hline DAS 28-CRP, median (p25-p75) & $3.37(2.11-4.4)$ & $3.17(2.09-4.2)$ & NS \\
\hline Dyslipidemia, n (\%) & $50(31.4)$ & $43(27)$ & NS \\
\hline Hypertension, n (\%) & $55(34.6)$ & $48(30.2)$ & NS \\
\hline Diabetes Mellitus, $n(\%)$ & $24(15.1)$ & $23(14.5)$ & NS \\
\hline Active smoking, $n(\%)$ & $8(5)$ & $8(5)$ & NS \\
\hline \multicolumn{4}{|l|}{ Seropositivity } \\
\hline Anti-CCP antibody positivity, n (\%) & $102(64.2)$ & $89(56)$ & 0.137 \\
\hline Anti-CCP antibody titers, median (p25-p75) & $97.1(1.7-198)$ & $8.5(1.1-145)$ & 0.003 \\
\hline IgG RF positivity, n (\%) & $31(19.5)$ & $24(15.1)$ & 0.299 \\
\hline IgG RF titers, median (p25-p75) & $5(2-13)$ & $4.9(2-13)$ & 0.529 \\
\hline IgM RF positivity, n (\%) & $136(85.5)$ & $126(79.2)$ & 0.141 \\
\hline IgM RF titers, median (p25-p75) & $198(41-200)$ & $177(28-200)$ & 0.067 \\
\hline IgA RF positivity, n (\%) & $98(61.6)$ & $92(57.9)$ & 0.493 \\
\hline IgA RF titers, median (p25-p75) & $52.9(9.3-193)$ & $33(5-159)$ & 0.060 \\
\hline
\end{tabular}

NS, non-significant; RA, rheumatoid arthritis; BMI, body mass index; BEI, biomass exposure index; DAS28-CRP, disease activity score using 28 joints-C-reactive protein; anti-CCP, anti-cyclic citrullinated peptide; RF, rheumatoid facto

\section{REFERENCES:}

[1] Fullerton DG, Bruce N, Gordon SB. Indoor air pollution from biomass fue smoke is a major health concern in the developing world. Trans R Soc Trop Med Hyg. 2008;102(9):843-51.

Disclosure of Interests: None declared

DOI: 10.1136/annrheumdis-2021-eular.2778

\section{POS0483 SUBCLINICAL ATHEROSCLEROSIS IN THE FIRST FIVE YEARS OF RHEUMATOID ARTHRITIS DIAGNOSIS}

N. Guajardo-Jauregui ${ }^{1}$, I. J. Colunga-Pedraza ${ }^{1}$, J. R. Azpiri-López ${ }^{2}$, D. Á. Galarza-Delgado ${ }^{1}$, A. B. Rodriguez-Romero ${ }^{1}$, S. Lugo-Perez ${ }^{2}$, A. Cárdenas ${ }^{1}$, A. C. Garza Acosta ${ }^{3} .{ }^{1}$ Hospital Universitario "Dr. José Eleuterio González", Universidad Autónoma de Nuevo León, Rheumatology, Monterrey, Mexico; ${ }^{2}$ Hospital Universitario “Dr. José Eleuterio González”, Universidad Autónoma de Nuevo León, Cardiology, Monterrey, Mexico; ${ }^{3}$ Hospital Universitario "Dr. José Eleuterio González”, Universidad Autónoma de Nuevo León, Radiology, Monterrey, Mexico

Background: Patients with rheumatoid arthritis (RA) have a higher risk of developing a cardiovascular (CV) event than the general population, due to an acceler ated process of atherosclerosis (1), which has been documented to begin in early stages of the disease and is directly associated with systemic inflammation (2).

Objectives: The aim of this study was to compare the prevalence of subclinical atherosclerosis detected by carotid ultrasound (US) in patients with RA in the first five years of diagnosis and healthy controls.

Methods: This was a cross-sectional, observational, and comparative study. A total of 53 patients aged 40-75 years old, with RA diagnosis, in the previous five years, according to the 2010 ACR/EULAR classification criteria and 53 controls matched by age $( \pm 5$ years), gender and comorbidities were included in this study. Subjects with a previous CV event, such as myocardia infarction, cerebrovascular event and peripheral arterial disease, another connective tissue disease and pregnant women were excluded from this study. A high-resolution B-mode carotid US was performed in all study subjects. Subclinical atherosclerosis was evaluated as the presence of carotid plaque (CP) or an increased carotid intima media thickness (cIMT). CP was defined as a cIMT $\geq 1.2 \mathrm{~mm}$ or a focal narrowing $\geq 0.5 \mathrm{~mm}$ of the surrounding lumen, and an increased cIMT was defined as a value $\geq 0.8 \mathrm{~mm}$. Distribution was evaluated with the Kolmogorov-Smirnov test. Comparisons were done with $\chi^{2}$ test and Fisher's exact test for qualitative variables, and Student's t test and Mann-Whitney's $U$ test for quantitative variables. A $p$-value $<0.05$ was considered statistically significant.

Results: Comparisons of demographic characteristics showed no differ ences between the RA group and the control group (Table 1). When comparing carotid US findings there was a difference in the presence of $\mathrm{CP}$, being more prevalent in RA patients $(26.4 \%$ vs $11.3 \%, p=0.047)$, in the presence of an increased cIMT, being more prevalent in RA patients $(34.0 \%$ vs $3.8 \%$, $p=<0.001$ ), in the cIMT as a quantitative variable, being higher in RA patients $(0.70 \mathrm{~mm}$ vs $0.59 \mathrm{~mm}, p=<0.001$ in the right carotid artery, and $0.75 \mathrm{~mm}$ vs 